\title{
Dark Atoms and the Positron-Annihilation-Line Excess in the Galactic Bulge
}

\author{
J.-R. Cudell, ${ }^{1}$ M. Yu. Khlopov, ${ }^{2,3,4}$ and Q. Wallemacq ${ }^{1}$ \\ ${ }^{1}$ IFPA, "Département d’AGO”, Université de Liège, Sart Tilman, 4000 Liège, Belgium \\ ${ }^{2}$ National Research Nuclear University "Moscow Engineering Physics Institute," Moscow 115409, Russia \\ ${ }^{3}$ Centre for Cosmoparticle Physics “Cosmion," 115409 Moscow, Russia \\ ${ }^{4}$ APC Laboratory 10, rue Alice Domon et Léonie Duquet, 75205 Paris Cedex 13, France
}

Correspondence should be addressed to Q. Wallemacq; quentin.wallemacq@ulg.ac.be

Received 25 November 2013; Accepted 13 January 2014; Published 25 February 2014

Academic Editor: Chris Kouvaris

Copyright (C) 2014 J.-R. Cudell et al. This is an open access article distributed under the Creative Commons Attribution License, which permits unrestricted use, distribution, and reproduction in any medium, provided the original work is properly cited. The publication of this article was funded by SCOAP S $^{3}$

It was recently proposed that stable particles of charge $-2, \mathrm{O}^{--}$, can exist and constitute dark matter after they bind with primordial helium in $\mathrm{O}$-helium $(\mathrm{OHe})$ atoms. We study here in detail the possibility that this model provides an explanation for the excess of gamma radiation in the positron-annihilation line from the galactic bulge observed by INTEGRAL. This explanation assumes that $\mathrm{OHe}$, excited to a $2 \mathrm{~s}$ state through collisions in the central part of the Galaxy, deexcites to its ground state via an $E 0$ transition, emitting an electron-positron pair. The cross-section for OHe collisions with excitation to $2 \mathrm{~s}$ level is calculated and it is shown that the rate of such excitations in the galactic bulge strongly depends not only on the mass of O-helium, which is determined by the mass of $\mathrm{O}^{--}$, but also on the density and velocity distribution of dark matter. Given the astrophysical uncertainties on these distributions, this mechanism constrains the $\mathrm{O}^{--}$mass to lie in two possible regions. One of these is reachable in the experimental searches for stable multicharged particles at the LHC.

\section{Introduction}

According to modern cosmology, dark matter corresponds to $25 \%$ of the total cosmological density, is nonbaryonic, and consists of new stable particles. Such particles (see [16] for reviews and references) should be stable, provide the measured dark-matter density, and be decoupled from plasma and radiation at least before the beginning of the matter-dominated era. It was recently shown that heavy stable particles of charge $-2, \mathrm{O}^{--}$, bound to primordial helium in $\mathrm{OHe}$ atoms, can provide an interesting explanation for cosmological dark matter $[6,7]$. It should also be noted that the nuclear cross-section of the O-helium interaction with matter escapes the severe constraints [8-10] on strongly interacting dark-matter particles (SIMPs) [8-16] imposed by the XQC experiment $[17,18]$.

The hypothesis of composite O-helium dark matter, first considered to provide a solution to the puzzles of direct darkmatter searches, can offer an explanation for another puzzle of modern astrophysics $[6,7,19]$ : this composite dark-matter model can explain the excess of gamma radiation in the electron-positron-annihilation line, observed by INTEGRAL in the galactic bulge (see [20] for a review and references). The explanation assumes that $\mathrm{OHe}$ provides all the galactic dark matter and that its collisions in the central part of the Galaxy result in 2s-level excitations of $\mathrm{OHe}$ which are deexcited to the ground state by an $E 0$ transition, in which an electron-positron pair is emitted. If the 2 s level is excited, pair production dominates over the two-photon channel in the deexcitation, because electrons are much lighter than helium nuclei, and positron production is not accompanied by a strong gamma-ray signal.

According to [21] the rate of positron production 3 . $10^{42} \mathrm{~s}^{-1}$ is sufficient to explain the excess in the positronannihilation line from the bulge measured by INTEGRAL. In the present paper we study the process of 2s-level excitation of $\mathrm{OHe}$ from collisions in the galactic bulge and determine the conditions under which such collisions can provide 
the observed excess. Inelastic interactions of O-helium with matter in interstellar space and subsequent deexcitation can give rise to radiation in the range from a few $\mathrm{keV}$ to a few $\mathrm{MeV}$. In the galactic bulge with radius $r_{b} \sim 1 \mathrm{kpc}$ the number density of O-helium can be of the order of $n_{o} \approx 3 \cdot 10^{-3} / S_{3} \mathrm{~cm}^{-3}$ or larger, and the collision rate of $\mathrm{O}$ helium in this central region was estimated in [19]: $d N / d t=$ $n_{o}^{2} \sigma v_{h} 4 \pi r_{b}^{3} / 3 \approx 3 \cdot 10^{42} S_{3}^{-2} \mathrm{~s}^{-1}$, with $S_{3}=m_{\mathrm{OHe}} / 1 \mathrm{TeV}$. At the velocity of $v_{h} \sim 3 \cdot 10^{7} \mathrm{~cm} / \mathrm{s}$ energy transfer in such collisions is $\Delta E \sim 1 \mathrm{MeVS}_{3}$. These collisions can lead to excitation of $\mathrm{O}$-helium. If OHe levels with nonzero angular momentum are excited, gamma lines should be observed from transitions $(n>m) E_{n m}=1.598 \mathrm{MeV}\left(1 / m^{2}-1 / n^{2}\right)$ (or from similar transitions corresponding to the case $I_{o}=1.287 \mathrm{MeV}$ ) at the level $3 \cdot 10^{-4} S_{3}^{-2}\left(\mathrm{~cm}^{2} \text { s MeV ster }\right)^{-1}$.

\section{Collisional Excitation Cross-Section}

The studied reaction is the collision between two OHe atoms, both being initially in their ground state $|1 \mathrm{~s}\rangle$, giving rise to the excitation of one of them to a $|n s\rangle$ state while the other remains in its ground state:

$$
\mathrm{OHe}(1 \mathrm{~s})+\mathrm{OHe}(1 \mathrm{~s}) \longrightarrow \mathrm{OHe}(1 \mathrm{~s})+\mathrm{OHe}(n \mathrm{~s})
$$

If we work in the rest frame of the OHe that gets excited and if we neglect its recoil after the collision, the differential cross-section of the process is given by

$$
\begin{aligned}
d \sigma(1 \mathrm{~s} \longrightarrow n \mathrm{~s})= & 2 \pi\left|\left\langle n \mathrm{~s}, \vec{p}^{\prime}|U| 1 \mathrm{~s}, \vec{p}\right\rangle\right|^{2} \\
& \times \delta\left(\frac{p^{\prime 2}}{2 M}+E_{n \mathrm{~s}}-\frac{p^{2}}{2 M}-E_{1 \mathrm{~s}}\right) \frac{d^{3} p^{\prime}}{(2 \pi)^{3}}
\end{aligned}
$$

where $M$ is the mass of OHe, $\vec{p}$ and $\vec{p}^{\prime}$ are the momenta of the incident $\mathrm{OHe}$ before and after the collision, $E_{1 \mathrm{~s}}$ and $E_{n \mathrm{~s}}$ are the ground-state and excited-state energies of the target $\mathrm{OHe}$, and $U$ is the interaction potential between the incident and the target OHe's.

We will neglect the internal structure of the incident $\mathrm{OHe}$, so that its wave functions are plane waves. $\psi_{\vec{p}}$ is normalized to obtain a unit incident current density and the normalisation of $\psi_{\vec{p}^{\prime}}$ is chosen for it to be pointlike, that is, the Fourier transform of $\delta^{(3)}(\vec{r})[22]$ :

$$
\begin{gathered}
\psi_{\vec{p}}=\sqrt{\frac{M}{p}} e^{i \vec{p} \cdot \vec{r}}, \\
\psi_{\vec{p}^{\prime}}=e^{i \vec{p}^{\prime} \cdot \vec{r}}
\end{gathered}
$$

where $\vec{r}$ is the position vector of the incident OHe and $p=|\vec{p}|$.

In the following, we will be led to consider $\mathrm{O}^{--}$masses which are much larger than the mass of helium or the boundstate energies. Therefore, the origin of the rest frame of the target $\mathrm{OHe}$ coincides with the position of its $\mathrm{O}^{--}$component and its reduced mass $\mu$ can be taken as the mass of helium $M_{\mathrm{He}}$.
The OHe that gets excited is described as a hydrogen-like atom, with energy levels $E_{n s}=-0.5 M_{\mathrm{He}}\left(Z_{\mathrm{He}} Z_{\mathrm{O}} \alpha\right)^{2} / n^{2}$ and initial and final bound-state wave functions $\psi_{1 \mathrm{~s}}$ and $\psi_{n \mathrm{~s}}$ of a hydrogenoid atom with a Bohr radius $a_{0}=\left(M_{\mathrm{He}} Z_{\mathrm{He}} Z_{\mathrm{O}} \alpha\right)^{-1}$.

The incident $\mathrm{OHe}$ interacts with the $\mathrm{O}^{--}$and helium components in the target $\mathrm{OHe}$, so that the interaction potential $U$ is the sum of the two contributions $U_{\mathrm{O}}$ and $U_{\mathrm{He}}$ :

$$
U(\vec{r})=U_{\mathrm{O}}(\vec{r})+U_{\mathrm{He}}\left(\vec{r}-\vec{r}_{\mathrm{He}}\right),
$$

where $\vec{r}_{\mathrm{He}}$ is the position vector of the helium component.

The first term $U_{\mathrm{O}}$ gives a zero contribution to the integral of expression (2) since the states $\psi_{1 \mathrm{~s}}$ and $\psi_{n \mathrm{~s}}$ are orthogonal. For the second term, we treat the incident $\mathrm{OHe}$ as a heavy neutron colliding on a helium nucleus through short-range nuclear forces. The interaction potential can then be written in the form of a contact term:

$$
U_{\mathrm{He}}\left(\vec{r}-\vec{r}_{\mathrm{He}}\right)=-\frac{2 \pi}{M_{\mathrm{He}}} a_{0} \delta\left(\vec{r}-\vec{r}_{\mathrm{He}}\right),
$$

where we have normalised the delta function to obtain an OHe-helium elastic cross-section equal to $4 \pi a_{0}^{2}$.

Going to spherical coordinates for $\vec{p}^{\prime}$ and integrating over $p^{\prime}=\left|\vec{p}^{\prime}\right|$ in the differential cross-section (2), together with the previous expressions (3), (4), and (5), we get

$$
\begin{aligned}
d \sigma(1 \mathrm{~s} \longrightarrow n \mathrm{~s})= & \left(\frac{M}{M_{\mathrm{He}}}\right)^{2} a_{0}^{2}\left(\frac{p^{\prime}}{p}\right) \\
& \times\left|\int e^{-i \vec{q} \cdot \vec{r}_{\mathrm{He}}} \psi_{n \mathrm{~s}}^{*} \psi_{1 \mathrm{~s}} d^{3} r_{\mathrm{He}}\right|^{2} d \Omega,
\end{aligned}
$$

where $\vec{q}=\vec{p}^{\prime}-\vec{p}$ is the transferred momentum and $d \Omega$ is the solid angle. From the integration over the delta function in (2), we have obtained the conservation of energy during the process:

$$
p^{\prime 2}=p^{2}+2 M\left(E_{1 \mathrm{~s}}-E_{n \mathrm{~s}}\right) .
$$

It leads to the threshold energy corresponding to $p^{\prime 2}=0$ and to a minimum incident velocity $v_{\min }=\sqrt{2\left(E_{n \mathrm{~s}}-E_{1 \mathrm{~s}}\right) / M}$. The previous expression for $p^{\prime}$ allows us to express the squared modulus of $\vec{q}$ as

$$
\begin{aligned}
q^{2}=2( & p^{2}+M\left(E_{1 \mathrm{~s}}-E_{n \mathrm{~s}}\right) \\
& \left.-p \sqrt{p^{2}+2 M\left(E_{1 \mathrm{~s}}-E_{n \mathrm{~s}}\right) \cos \theta}\right),
\end{aligned}
$$

where $\theta$ is the deviation angle of the incident $\mathrm{OHe}$ with respect to the collision axis in the rest frame of the target $\mathrm{OHe}$.

$e^{+} e^{-}$pairs will be dominantly produced if $\mathrm{OHe}$ is excited to a $2 s$ state, since the only deexcitation channel is in this case from $2 \mathrm{~s}$ to $1 \mathrm{~s}$. As $e^{+} e^{-}$pair production is the only possible channel, the differential pair-production cross-section $d \sigma_{e e}$ is equal to the differential collisional excitation cross-section. By particularizing expression (6) to the case $n=2$, one finally gets

$$
\frac{d \sigma_{e e}}{d \cos \theta}=512^{2}\left(\frac{2 \pi M^{2}}{M_{\mathrm{He}}^{2}}\right) a_{0}^{6}\left(\frac{p^{\prime}}{p}\right) \frac{q^{4}}{2\left(4 a_{0}^{2} q^{2}+9\right)^{6}} .
$$




\section{The $e^{+} e^{-}$Pair-Production Rate in the Galactic Bulge}

The total $e^{+} e^{-}$pair-production rate in the galactic bulge is given by

$$
\left.\frac{d N}{d t}\right|_{e e}=\int_{V_{b}} \frac{\rho_{\mathrm{DM}}^{2}(\vec{R})}{M^{2}}\left\langle\sigma_{e e} v\right\rangle(\vec{R}) d \vec{R},
$$

where $V_{b}$ is the volume of the galactic bulge, which is a sphere of radius $R_{b}=1.5 \mathrm{kpc}, \rho_{\mathrm{DM}}$ is the energy density distribution of dark matter in the galactic halo, and $\left\langle\sigma_{e e} v\right\rangle$ is the pairproduction cross-section $\sigma_{e e}$ times relative velocity $v$ averaged over the velocity distribution of dark-matter particles. The total pair-production cross-section $\sigma_{e e}$ is obtained by integrating (9) over the diffusion angle. Its dependence on the relative velocity $v$ is contained in $p, p^{\prime}$, and $q$ through $p=M v$ and the expressions (7) and (8) of $p^{\prime}$ and $q$ in terms of $p$.

We use a Burkert $[23,24]$ flat, cored, dark-matter density profile known to reproduce well the kinematics of disk systems in massive spiral galaxies and supported by recent simulations including supernova feedback and radiation pressure of massive stars [25] in response to the cuspy halo problem:

$$
\rho_{\mathrm{DM}}(R)=\rho_{0} \frac{R_{0}^{3}}{\left(R+R_{0}\right)\left(R^{2}+R_{0}^{2}\right)},
$$

where $R$ is the distance from the galactic center. The central dark-matter density $\rho_{0}$ is left as a free parameter and $R_{0}$ is determined by requiring that the local dark-matter density at $R=R_{\odot}=8 \mathrm{kpc}$ is $\rho_{\odot}=0.3 \mathrm{GeV} / \mathrm{cm}^{3}$. The dark-matter mass enclosed in a sphere of radius $R$ is therefore given by

$$
\begin{aligned}
M_{\mathrm{DM}}(R)=\rho_{0} \pi R_{0}^{3}\{ & \log \left(\frac{R^{2}+R_{0}^{2}}{R_{0}^{2}}\right) \\
& \left.+2 \log \left(\frac{R+R_{0}}{R_{0}}\right)-2 \arctan \left(\frac{R}{R_{0}}\right)\right\} .
\end{aligned}
$$

For the baryons in the bulge, we use an exponential profile [26] of the form

$$
\rho_{b}(R)=\frac{M_{\text {bulge }}}{8 \pi R_{b}^{3}} e^{-R / R_{b}},
$$

where $M_{\text {bulge }}=10^{10} M_{\odot}$ [27] is the mass of the bulge. This gives the baryonic mass distribution in the galactic bulge

$$
M_{b}(R)=M_{\text {bulge }}\left\{1-e^{-R / R_{b}}\left(1+\frac{R}{R_{b}}+\frac{R^{2}}{R_{b}^{2}}\right)\right\} .
$$

We assume a Maxwell-Boltzmann velocity distribution for the dark-matter particles of the galactic halo, with a velocity dispersion $u(R)$ and a cutoff at the galactic escape velocity $v_{\text {esc }}(R)$ :

$$
f\left(R, \vec{v}_{h}\right)=\frac{1}{C(R)} e^{-v_{h}^{2} / u^{2}(R)},
$$

where $\vec{v}_{h}$ is the velocity of the dark-matter particles in the frame of the halo and $C(R)=\pi u^{2}\left(\sqrt{\pi} u \operatorname{erf}\left(v_{\text {esc }} / u\right)-\right.$ $\left.2 v_{\text {esc }} e^{-v_{\text {esc }}^{2} / u^{2}}\right)$ is a normalization constant such that $\int_{0}^{v_{\text {esc }}(R)} f\left(R, \vec{v}_{h}\right) d \vec{v}_{h}=1$.

The radial dependence of the velocity dispersion is obtained via the virial theorem:

$$
u(R)=\sqrt{\frac{G M_{\mathrm{tot}}(R)}{R}},
$$

where $M_{\text {tot }}=M_{\mathrm{DM}}+M_{b}$, while $v_{\mathrm{esc}}=\sqrt{2} u$.

Using the velocity distribution (15), going to center-ofmass and relative velocities $\vec{v}_{\mathrm{CM}}$ and $\vec{v}$, and performing the integrals over $\vec{v}_{\mathrm{CM}}$, we obtain for the mean pair-production cross-section times relative velocity

$$
\begin{aligned}
\left\langle\sigma_{e e} v\right\rangle= & \frac{1}{u^{2}} \frac{\sqrt{2 \pi} u \operatorname{erf}\left(\sqrt{2} v_{\mathrm{esc}} / u\right)-4 v_{\mathrm{esc}} e^{-2 v_{\mathrm{esc}}^{2} / u^{2}}}{\left(\sqrt{\pi} u \operatorname{erf}\left(v_{\mathrm{esc}} / u\right)-2 v_{\mathrm{esc}} e^{-v_{\mathrm{esc}}^{2} / u^{2}}\right)^{2}} \\
& \times \int_{0}^{2 v_{\mathrm{esc}}} \sigma_{e e}(v) v^{3} e^{-v^{2} / 2 u^{2}} d v,
\end{aligned}
$$

which is also a function of $R$ through $u$ and $v_{\text {esc }}$. Putting (9), (11), (12), (14), (16), and (17) together allows us to compute the pair-production rate in the galactic bulge defined in (10) as a function of $\rho_{0}$ and $M$.

\section{Results}

The rate of excessive $e^{+} e^{-}$pairs to be generated in the galactic bulge was estimated in [21] to be $d N /\left.d t\right|_{\text {obs }}=3 \times 10^{42} \mathrm{~s}^{-1}$. We computed $d N /\left.d t\right|_{e e}$ for a large range of central darkmatter densities, going from $0.3 \mathrm{GeV} / \mathrm{cm}^{3}$ to an ultimate upper limit of $10^{4} \mathrm{GeV} / \mathrm{cm}^{3}$ [28]. For each value of $\rho_{0}$, we searched for the mass $M$ of $\mathrm{OHe}$ that reproduces the observed rate. The results are shown in Figure 1.

The observed rate can be reproduced from a value of $\rho_{0} \simeq 115 \mathrm{GeV} / \mathrm{cm}^{3}$, corresponding to an OHe mass of $M \simeq$ $1.25 \mathrm{TeV}$. As $\rho_{0}$ gets larger, two values of $M$ are possible, with the lower one going from $1.25 \mathrm{TeV}$ to $130 \mathrm{GeV}$ and the upper one going from 1.25 to $130 \mathrm{TeV}$ as $\rho_{0}$ goes from 115 to $10^{4} \mathrm{GeV} / \mathrm{cm}^{3}$.

\section{Conclusion}

The existence of heavy stable particles is one of the most popular solutions for the dark- matter problem. Usually they are considered to be electrically neutral. But dark matter can potentially be made of stable heavy charged particles bound in neutral atom-like states by Coulomb attraction. An analysis of the cosmological data and of the atomic composition of the Universe forces the particle to have charge -2 . $\mathrm{O}^{--}$is then trapped by primordial helium in neutral $\mathrm{O}-$ helium states and this avoids the problem of overproduction of anomalous isotopes, which are severely constrained by observations. Here we have shown that the cosmological model of O-helium dark matter can explain the puzzle of positron line emission from the center of our Galaxy. 


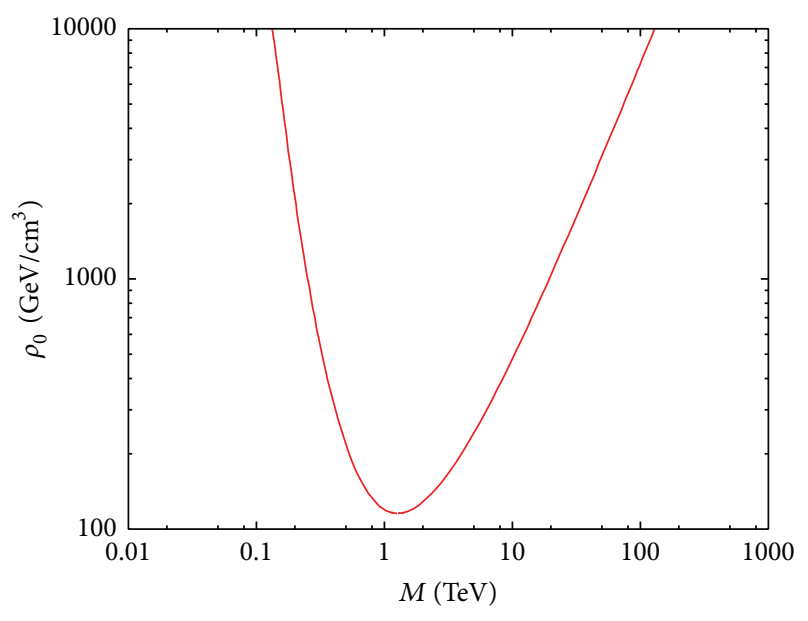

Figure 1: Values of the central dark-matter density $\rho_{0}\left(\mathrm{GeV} / \mathrm{cm}^{3}\right)$ and of the OHe mass $M(\mathrm{TeV})$ reproducing the excess of $e^{+} e^{-}$pairs production in the galactic bulge. Below the red curve, the predicted rate is too low.

The proposed explanation is based on the assumption that $\mathrm{OHe}$ dominates the dark-matter sector. Its collisions can lead to E0 deexcitations of the 2 s states excited by the collisions. The estimated luminosity in the electron-positronannihilation line strongly depends not only on the mass of $\mathrm{O}^{--}$but also on the density profile and velocity distribution of dark matter in the galactic bulge. Note that the density profile we considered is used only to obtain a reasonable estimate for the uncertainties on the density in the bulge. It indeed underestimates the mass of the Galaxy, but it shows that the uncertainties on the astrophysical parameters are large enough to reproduce the observed excess for a rather wide range of masses of $\mathrm{O}^{--}$. For a fixed density profile and a fixed velocity distribution, only two values of the $\mathrm{O}^{--}$mass lead to the necessary rate of positron production. The lower value of this mass, which does not exceed $1.25 \mathrm{TeV}$, is within the reach of experimental searches for multicharged stable heavy particles at the LHC.

\section{Conflict of Interests}

The authors declare that there is no conflict of interests regarding the publication of this paper.

\section{Acknowledgment}

The authors express their gratitude to A. S. Romaniouk for discussions.

\section{References}

[1] M. Yu. Khlopov, Cosmoparticle Physics, World Scientific, Singapore, 1999.

[2] M. Yu. Khlopov, "Cosmoarcheology. Direct and indirect astrophysical effects of hypothetical particles and fields," in Cosmion94, M. Yu. Khlopov, M. E. Prokhorov, A. A. Starobinsky, and J.
Tran Thanh Van, Eds., pp. 67-76, Editions Frontières, Quebec, Canada, 1996.

[3] M. Y. Khlopov, "Proceedings to the 9th workshop 'what comes beyond the standard models"' Bled Workshops in Physics, vol. 7, no. 2, p. 51, 2006.

[4] M. Y. Khlopov, "Proceedings to the 10th workshop 'what comes beyond the standard models"' Bled Workshops in Physics, vol. 8, no. 2, p. 114, 2007.

[5] M. Yu. Khlopov, Fundamentals of Cosmoparticle Physics, CISPSpringer, Cambridge, UK, 2012.

[6] M. Yu. Khlopov, "Fundamental particle structure in the cosmological dark matter," International Journal of Modern Physics A, vol. 28, no. 29, Article ID 1330042, 60 pages, 2013.

[7] M. Yu. Khlopov, "Physics of dark matter in the light of dark atoms," Modern Physics Letters A, vol. 26, no. 38, Article ID 2823, 2011.

[8] B. D. Wandelt, R. Dave, G. R. Farrar, P. C. McGuire, D. N. Spergel, and P. J. Steinhardt, "Self-interacting dark matter," http://arxiv.org/abs/astro-ph/0006344.

[9] P. C. McGuire and P. J. Steinhardt, "Cracking open the window for strongly interacting massive particles as the halo dark matter," http://arxiv.org/abs/astro-ph/0105567.

[10] G. Zaharijas and G. R. Farrar, "Window in the dark matter exclusion limits," Physical Review D, vol. 72, no. 8, Article ID 083502, 11 pages, 2005.

[11] C. B. Dover et al., "Cosmological constraints on new stable hadrons," Physical Review Letters, vol. 42, no. 17, pp. 1117-1120, 1979.

[12] S. Wolfram, "Abundances of new stable particles produced in the early universe," Physics Letters B, vol. 82, no. 1, pp. 65-68, 1979.

[13] G. D. Starkman, A. Gould, R. Esmailzadeh, and S. Dimopoulos, "Opening the window on strongly interacting dark matter," Physical Review D, vol. 41, no. 12, pp. 3594-3603, 1990.

[14] D. Javorsek, D. Elmore, E. Fischbach et al., "New experimental limits on strongly interacting massive particles at the TeV scale," Physical Review Letters, vol. 87, no. 23, Article ID 231804, 2001.

[15] S. Mitra, "Uranus's anomalously low excess heat constrains strongly interacting dark matter," Physical Review D, vol. 70, no. 10, Article ID 103517, 2004.

[16] G. D. Mack, J. F. Beacom, and G. Bertone, "Towards closing the window on strongly interacting dark matter: far-reaching constraints from Earth's heat flow," Physical Review D, vol. 76, no. 4, Article ID 043523, 2007.

[17] D. McCammon, R. Almy, S. Deiker et al., "A sounding rocket payload for X-ray astronomy employing high-resolution microcalorimeters," Nuclear Instruments and Methods in Physics Research Section A, vol. 370, no. 1, pp. 266-268, 1996.

[18] D. McCammon, R. Almy, E. Apodaca et al., "A high spectral resolution observation of the soft X-ray diffuse background with thermal detectors "' The Astrophysical Journal, vol. 576, no. 1, p. 188, 2002.

[19] M. Yu. Khlopov, "Composite dark matter from stable charged constituents," http://arxiv.org/abs/0806.3581.

[20] B. J. Teegarden, K. Watanabe, P. Jean et al., "INTEGRAL SPI limits on electron-positron annihilation radiation from the galactic plane," The Astrophysical Journal, vol. 621, no. 1, p. 296, 2005.

[21] D. P. Finkbeiner and N. Weiner, "Exciting dark matter and the INTEGRAL/SPI $511 \mathrm{keV}$ signal," Physical Review D, vol. 76, no. 8, Article ID 083519, 2007. 
[22] L. D. Landau and E. M. Lifshitz, Quantum Mechanics, Pergamon Press, Elmsford, NY, USA, 1965.

[23] A. Burkert, "The structure of dark matter haloes in dwarf galaxies," IAU Symposia, vol. 171, p. 175, 1996.

[24] A. Burkert, "The structure of dark matter haloes in dwarf galaxies," The Astrophysical Journal, vol. 447, no. 1, p. L25, 1995.

[25] A. V. Maccio, G. Stinson, C. B. Brook et al., "HALO Expansion in cosmological hydro simulations: toward a baryonic solution of the cusp/core problem in massive spirals," The Astrophysical Journal Letters, vol. 744, no. 1, p. L9, 2012.

[26] O. Y. Gnedin, A. V. Kravtsov, A. A. Klypin, and D. Nagai, "Response of dark matter halos to condensation of Baryons: cosmological simulations and improved adiabatic contraction model," The Astrophysical Journal, vol. 616, no. 1, p. 16, 2004.

[27] H. Mo, F. van den Bosch, and S. White, Galaxy Formation and Evolution, Cambridge University Press, Cambridge, UK, 2010.

[28] X. Hernandez and W. H. Lee, "An upper limit to the central density of dark matter haloes from consistency with the presence of massive central black holes," Monthly Notices of the Royal Astronomical Society, vol. 404, no. 1, p. L10, 2010. 

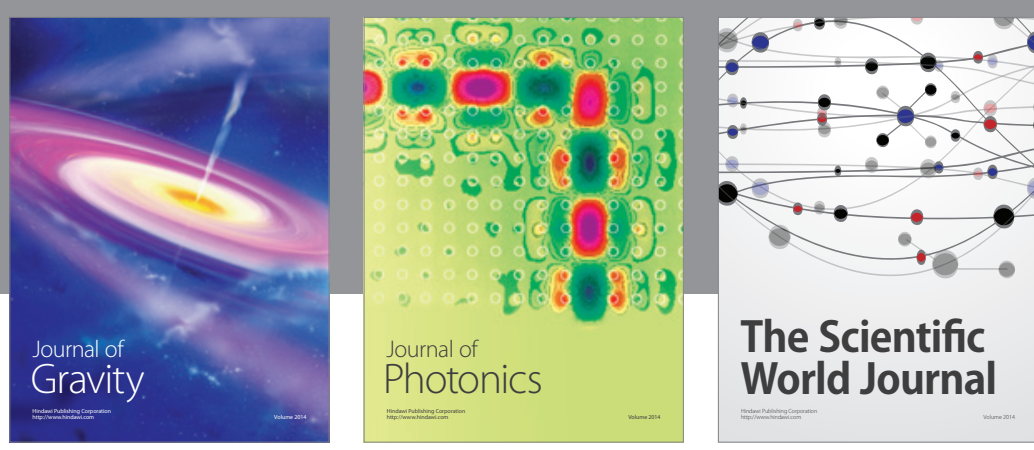

The Scientific World Journal
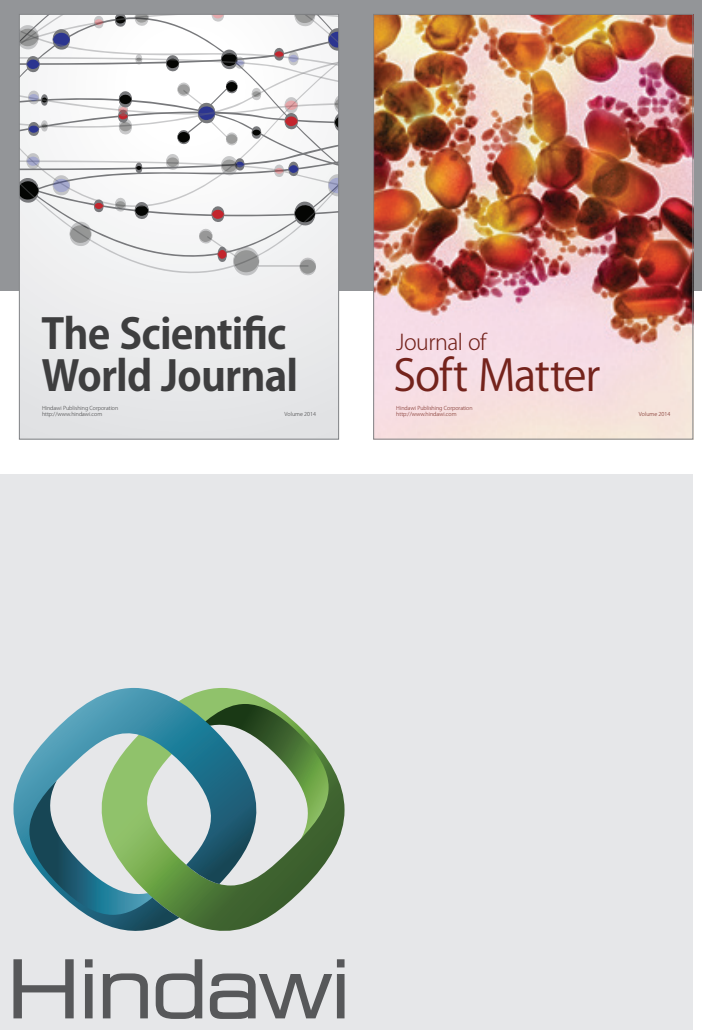

Submit your manuscripts at

http://www.hindawi.com

nternational Journal of

Statistical Mechanics
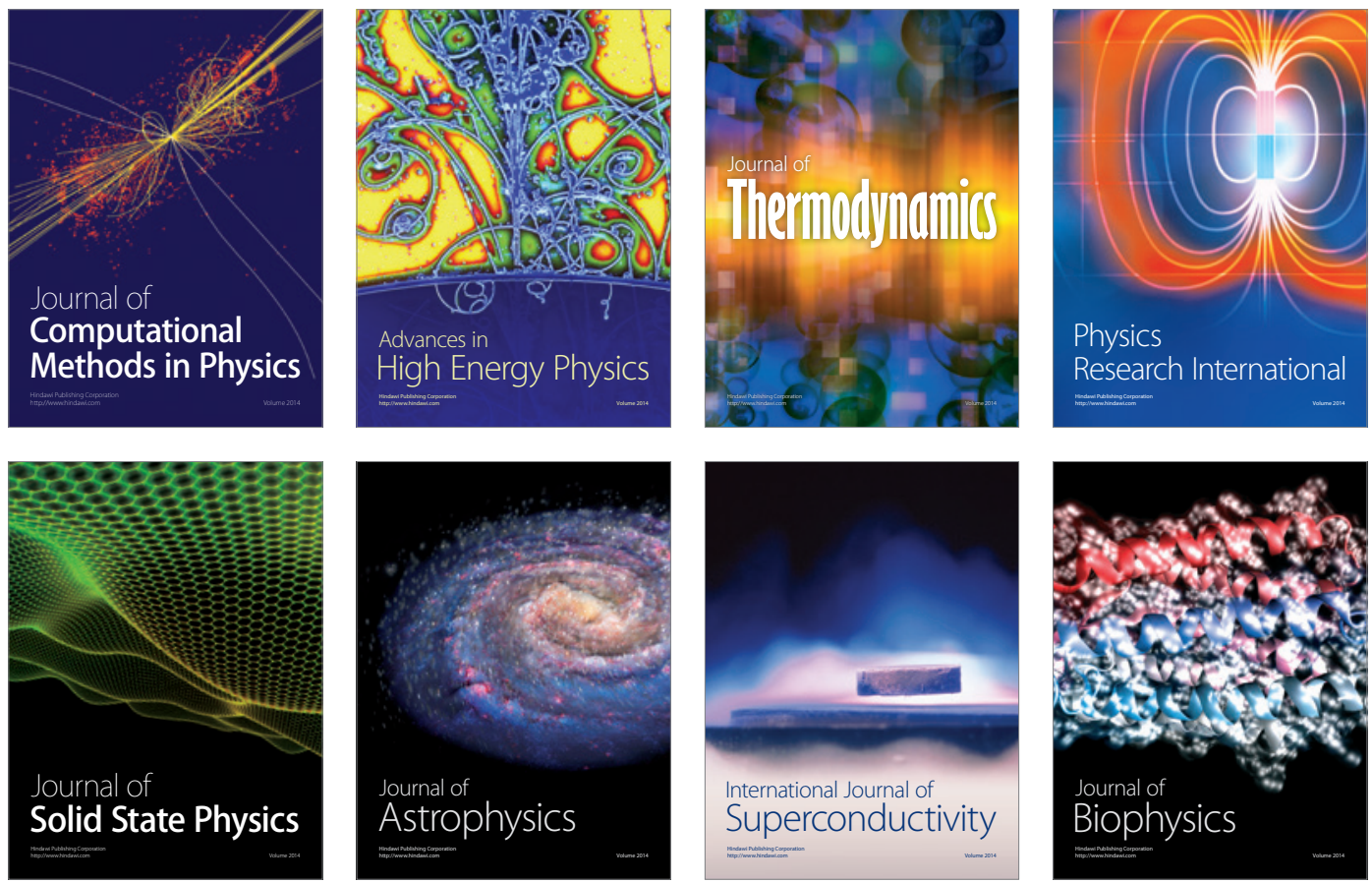
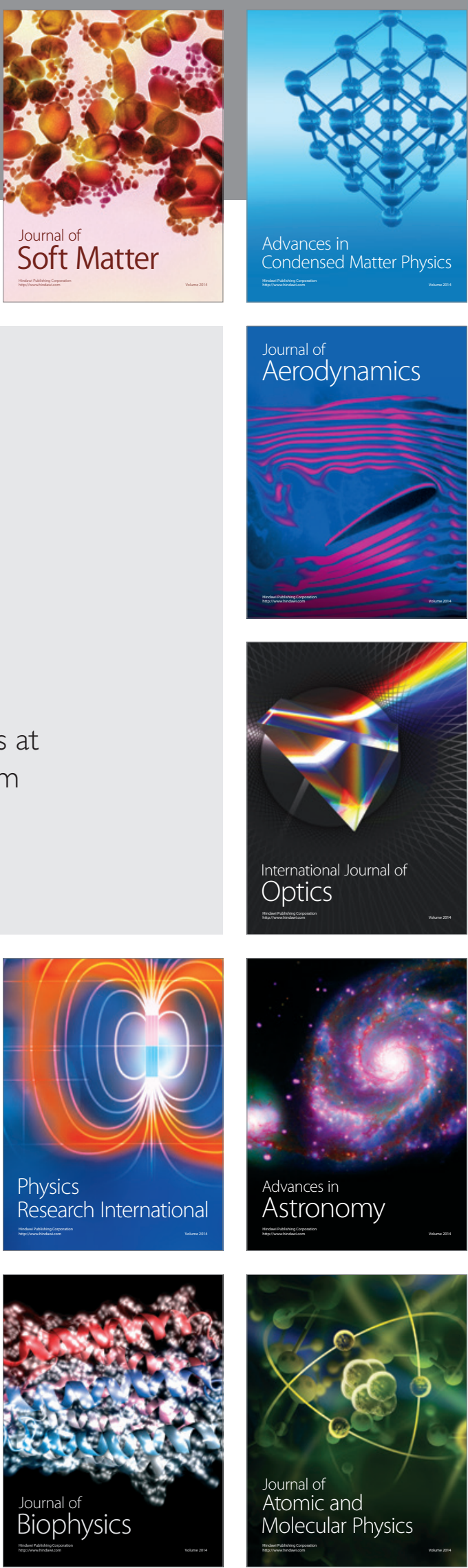